\title{
Intermediate Progenitors Facilitate Intracortical Progression of Thalamocortical Axons and Interneurons through CXCL12 Chemokine Signaling
}

\author{
Philipp Abe, ${ }^{1}$ Zoltán Molnár, ${ }^{2}$ Yi-Shiuan Tzeng, ${ }^{3}$ Dar-Ming Lai, ${ }^{4}$ Sebastian J. Arnold, $, 5,6$ and $\odot$ Ralf Stumm ${ }^{1}$ \\ ${ }^{1}$ Institute of Pharmacology and Toxicology, Jena University Hospital, Friedrich Schiller University Jena, 07747 Jena, Germany, ${ }^{2}$ Department of Physiology, \\ Anatomy and Genetics, University of Oxford, Oxford OX1 3QX, United Kingdom, ${ }^{3}$ Graduate Institute of Oncology, National Taiwan University, Taipei \\ 10002, Taiwan, ${ }^{4}$ Department of Surgery, National Taiwan University Hospital and National Taiwan University College of Medicine, Taipei 10002, Taiwan, \\ ${ }^{5}$ Renal Division, University Hospital Freiburg, 79106 Freiburg, Germany, and ${ }^{6}$ BIOSS Centre of Biological Signalling Studies and ${ }^{7}$ Institute of Experimental \\ and Clinical Pharmacology and Toxicology, Albert-Ludwigs-University, 79104 Freiburg, Germany
}

Glutamatergic principal neurons, GABAergic interneurons and thalamocortical axons (TCAs) are essential elements of the cerebrocortical network. Principal neurons originate locally from radial glia and intermediate progenitors (IPCs), whereas interneurons and TCAs are of extrinsic origin. Little is known how the assembly of these elements is coordinated. C-X-C motif chemokine 12 (CXCL12), which is known to guide axons outside the neural tube and interneurons in the cortex, is expressed in the meninges and IPCs. Using mouse genetics, we dissected the influence of IPC-derived CXCL12 on TCAs and interneurons by showing that $C x c l 12$ ablation in IPCs, leaving meningeal $C x c l 12$ intact, attenuates intracortical TCA growth and disrupts tangential interneuron migration in the subventricular zone. In accordance with strong CXCR4 expression in the forming thalamus and TCAs, we identified a CXCR4-dependent growth-promoting effect of CXCL12 on TCAs in thalamus explants. Together, our findings indicate a cell-autonomous role of CXCR4 in promoting TCA growth. We propose that CXCL12 signals from IPCs link cortical neurogenesis to the progression of TCAs and interneurons spatially and temporally.

Key words: cortical development; CXCL12/CXCR4; intermediate/basal progenitors; interneuron; thalamocortical axons; thalamus

Significance Statement

The cerebral cortex exerts higher brain functions including perceptual and emotional processing. Evolutionary expansion of the mammalian cortex is mediated by intermediate progenitors, transient amplifying cells generating cortical excitatory neurons. During the peak period of cortical neurogenesis, migrating precursors of inhibitory interneurons originating in subcortical areas and thalamic axons invade the cortex. Although defects in the assembly of cortical network elements cause neurological and mental disorders, little is known how neurogenesis, interneuron recruitment, and axonal ingrowth are coordinated. We demonstrate that intermediate progenitors release the chemotactic cytokine CXCL12 to promote intracortical interneuron migration and growth of thalamic axons via the cognate receptor CXCR4. This paracrine signal may ensure thalamocortical connectivity and dispersion of inhibitory neurons in the rapidly growing cortex.

\section{Introduction}

The cerebral cortex exerts perceptual, motor, and cognitive functions including emotional processing. Cortical neurons approx-

Received April 16, 2015; revised July 30, 2015; accepted Aug. 13, 2015.

Author contributions: P.A. and R.S. designed research; P.A. and Z.M. performed research; Y.-S.T., D.-M.L., and S.J.A. contributed unpublished reagents/analytic tools; P.A. and R.S. analyzed data; P.A., S.J.A., and R.S. wrote the paper.

The work was supported by DFG Grants STU 295/7-1 and AR732/1-1. We thank C. Anders, H. Bechmann, S. Bechmann, and H. Stadler for excellent technical assistance; Drs. Falko Nagel and Stefan Schulz for providing UMB-2 antibody; Dr. Navneet Vasistha (University of Oxford) for scientific support; Dr. Fritz G. Rathjen (Max Delbrück Center for Molecular Medicine) for L1 antibody; and Dr Tomomi Shimogori (RIKEN Brain Science Institute) for the Gbx2 probe. imately consist of $80 \%$ glutamatergic principal neurons (PNs) and 20\% GABAergic interneurons (Parnavelas, 2000). Thalamocortical axons (TCAs) terminating on layer IV PNs convey sensory information to the cortex and represent its major excitatory input. Neurodevelopmental defects that disturb the balance of these excitatory and inhibitory systems are thought to underlie epilepsy and mental disorders including schizophrenia, person-

\section{The authors declare no competing financial interests.}

Correspondence should be addressed to Dr RalfStumm, Institute of Pharmacology and Toxicology, Drackendorfer Strasse 1, 07747 Jena, Germany. E-mail: Ralf.Stumm@med.uni-jena.de.

DOI:10.1523/JNEUROSCI.1488-15.2015

Copyright $\odot 2015$ the authors $\quad$ 0270-6474/15/3513053-11 $\$ 15.00 / 0$ 
ality disorders, and autism spectrum disorders. Thus, elucidating the mechanisms that govern the assembly of the cortical network is central to understanding causes and pathogenesis of mental illnesses (Rubenstein, 2011).

Cortical PNs are generated locally, whereas cortical interneurons are of subpallial origin. In mice, most interneurons enter the cortex after embryonic day (E)12. They migrate preferentially on characteristic routes including the cortical subventricular zone (SVZ) and marginal zone (MZ) (Wonders and Anderson, 2006). Individual interneurons spend days migrating in the cortex and perform random movements. This unusual behavior is thought to disperse the cells before they integrate into the network (López-Bendito et al., 2008; Tanaka et al., 2009). Like interneurons, TCAs travel long distances before reaching their destination. Murine TCAs navigate through the ventral telencephalon for $2 \mathrm{~d}$ and reach the cortex $\sim$ E14. Until E15.5, they traverse the cortex in the SVZ and intermediate zone (IZ) to form contacts with the subplate, where they pause for several days before growing into the cortical plate (CP) (López-Bendito and Molnár, 2003; Mire et al., 2012). Thus, the peak of cortical neurogenesis, taking place from E12 to E16 in mice (Hevner et al., 2004), coincides with intracortical TCA growth and interneuron dispersion.

Cortical basal/intermediate progenitor cells (IPCs) generate a significant proportion of PN (Kowalczyk et al., 2009; Vasistha et al., 2014) and enable evolutionary expansion of the cortex by amplification of cell numbers (Martínez-Cerdeño et al., 2006). Sufficient interneurons and TCAs have to be integrated into the fast growing cortex, demanding signal mechanisms that coordinate PN production with progression of invading elements. Several findings suggest that the chemokine CXCL12 (stromal cell-derived factor-1) via its receptors CXCR4 and CXCR7 acts as such a coordinating signal. First, in addition to the meninges, CXCL12 is highly expressed in IPCs and guides migrating interneurons in the cortex (Stumm et al., 2003; Tiveron et al., 2006; Stumm and Hollt, 2007; Li et al., 2008; Lysko et al., 2011; Sánchez-Alcañiz et al., 2011; Wang et al., 2011; Abe et al., 2014). Second, we observed that CXCR4 is highly expressed in the forming thalamus and TCAs. In analogy to the CXCL12/CXCR4 axis de-

fining the initial trajectories of motor axons growing out of the neural tube (Lieberam et al., 2005), CXCL12 might similarly be involved in the formation of the thalamocortical projection.

Despite the well documented role of CXCL12 in cortical development, the specific contribution of IPC-derived CXCL12 has not been dissected. In this report, we used explant experiments and a wealth of histochemical and fluorescent dye labeling technology in a series of mouse genetic models including conditional Cxcl12 ablation in IPCs. We thus provide conclusive evidence
Table 1. Primary antibodies

\begin{tabular}{lll}
\hline Primary antibody & Concentration & Source \\
\hline Rabbit anti-GFP & $1: 500$ & Synaptic Systems \#132002 \\
Chicken anti-GFP & $1: 500$ & Aves Labs \#GFP-1020 \\
Chicken anti-neurofilament & 1:10,000; enhanced* & Aves Labs \#NFM (160 kDa) \\
Goat anti-SOX2 & 1:200 & R\&D Systems \#AF2018 \\
Rabbit anti-L1 & 1:400 & provided by Dr Fritz Rathjen (Berlin) \\
Rat anti-CXCR4 (2B11) & 1:1000; enhanced* & BD Bioscience \#551968 \\
Rabbit anti-CXCR4 (UMB-2) & 1:2; enhanced* & provided by Dr Stefan Schulz (Jena) \\
\hline
\end{tabular}

*Biotin-tyramine amplification.

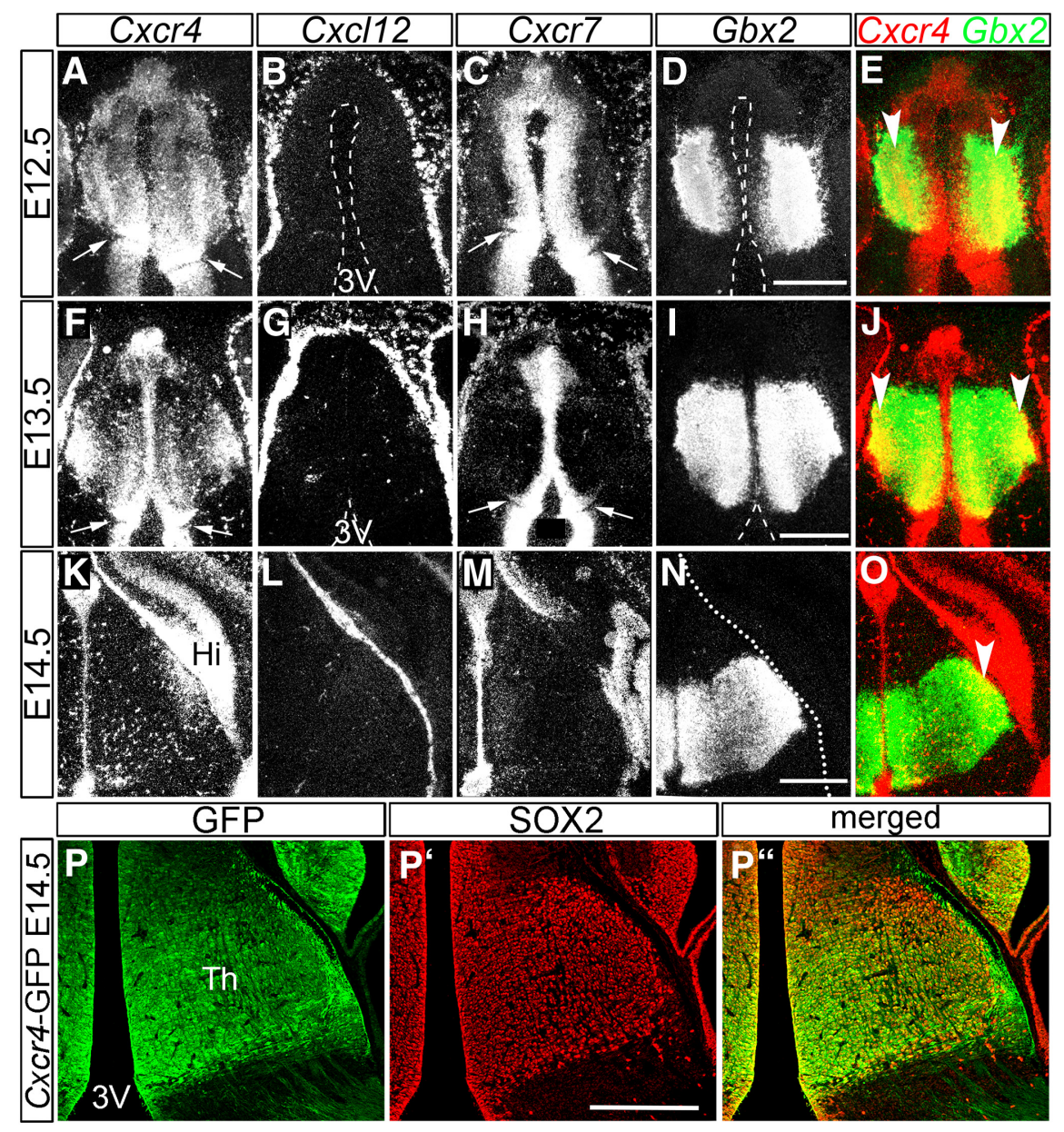

Figure 1. Patterns of $\mathrm{C} x \mathrm{Cr} 4, \mathrm{C} x \mathrm{Cr}$, and $\mathrm{Cx} / 12$ in the early thalamus. $\mathbf{A - 0}$, Darkfield micrographs of emulsion-dipped coronal sections show the thalamus after in situ hybridization with ${ }^{35}$ S-labeled probes for $C x c r 4(A, F, K), C x C 112(B, G, L), C X c r 7(C, H, M)$, and $\operatorname{Gbx2}(\boldsymbol{D}, \boldsymbol{I}, \boldsymbol{N})$ at E12.5 $(\boldsymbol{A}-\boldsymbol{E}), \mathrm{E} 13.5(\boldsymbol{F}-\boldsymbol{J})$, and E14.5 $(\boldsymbol{K}-\mathbf{O}) . \boldsymbol{E}, \boldsymbol{J}, \mathbf{O}$, Overlays of false color displays of the adjacent sections shown in $A, D ; F, I ;$ and $\boldsymbol{K}, \boldsymbol{N} . \boldsymbol{A}, \boldsymbol{E}, \boldsymbol{F}, J, \boldsymbol{K}, \boldsymbol{O}, \boldsymbol{C} \times(r)$ is expressed in the progenitor domain along the midline and in the $G b \times 2^{+}$ differentiation area of the thalamus. Arrowheads in $\boldsymbol{E}$, $\boldsymbol{J}$, and $\boldsymbol{O}$ point to $C x c r 4$-expressing lateral thalamic nuclei. $\boldsymbol{B}, \boldsymbol{G}, \boldsymbol{L}, \boldsymbol{C x} / 12$ is present in the meninges covering the thalamus. $C, H, M, C X C r 7$ is expressed in the progenitor domain along the midline. $A, C, F, H$, Arrows point to the zona limitans intrathalamica for orientation. $\boldsymbol{P}-\boldsymbol{P}^{\prime \prime}$, Confocal images show immunostained GFP and SOX2 in a coronal section of an E14.5 Cxcr4-GFP reporter mouse. 3V, Third ventricle; Hi, hippocampal anlage; Th, thalamus. Scale bars:D, $\mathbf{I}, \mathbf{N}$, $P^{\prime}, 500 \mu \mathrm{m}$. that CXCL12, emanating from IPCs, promotes intracortical TCA growth and interneuron migration in the SVZ.

\section{Materials and Methods}

Animals. Animal procedures were in accordance with German and EU guidelines. Cxcr4-GFP and CXCL12-RFP mice (Bhattacharyya et al., 2008) were on CD1 background; Cxcl12 ${ }^{+/-}$(Nagasawa et al., 1996), Cxcl12 $^{\text {LoxP }}$ (Tzeng et al., 2011), and Tbr2 ${ }^{+/ C r e}$ mice (Costello et al., 2011) 

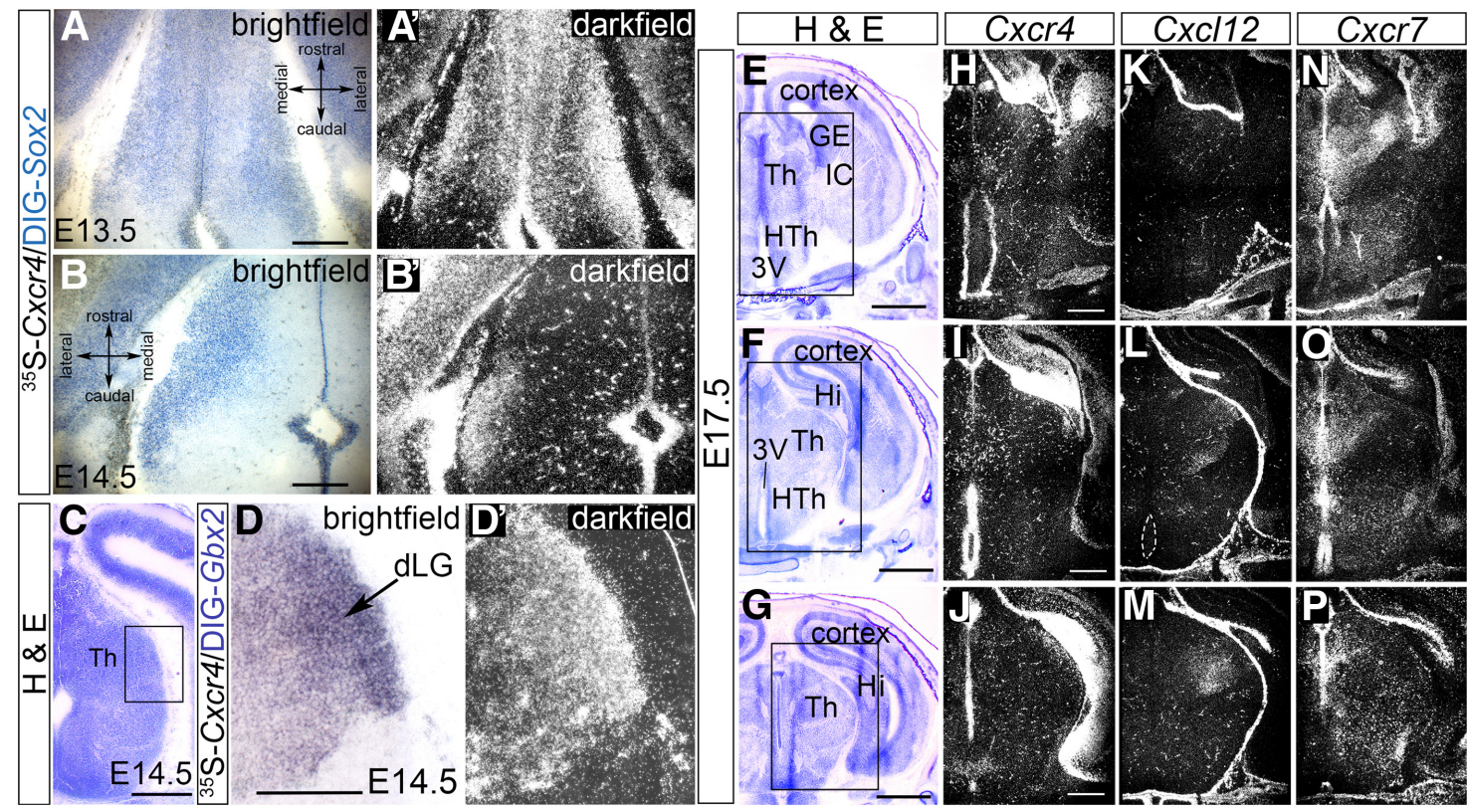

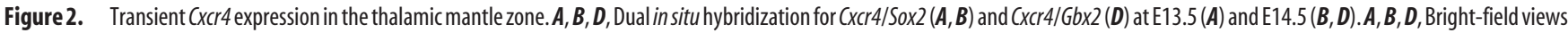
and $\boldsymbol{A}^{\prime}, \boldsymbol{B}^{\prime}, \boldsymbol{D}^{\prime}$ are dark-field views of the same specimens. $\boldsymbol{A}, \boldsymbol{B}$, Horizontal and $\boldsymbol{D}$ is a coronal section. (Xcr4 was detected with a ${ }^{35} S$-labeled probe (white signal in $\boldsymbol{A}^{\prime}, \boldsymbol{B}^{\prime}, \boldsymbol{D}^{\prime}$ ); Sox2 and Gbx2 were detected with digoxigenin (DIG)-labeled probes $\left(\boldsymbol{A}, \boldsymbol{B}, \boldsymbol{D}\right.$, blue signal). . Xcr 4 is expressed in the Sox ${ }^{+}$and $G b \times 2^{+}$thalamic mantle zone. $\boldsymbol{C}$, The H\&E-stained coronal section shows the diencephalon, the box corresponds to the thalamic area shown in $\boldsymbol{D}$. $\boldsymbol{E}-\boldsymbol{G}$, Boxes in the H\&E-stained coronal sections correspond to the thalamic area shown in $\boldsymbol{H}-\boldsymbol{P}$. Images show the thalamus at rostral (top), mid

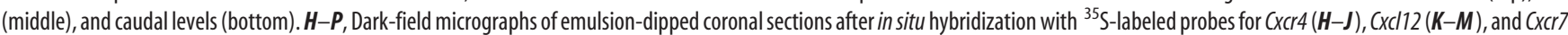
(N-P). 3V, Third ventricle; IC, internal capsule; dLG, dorsal lateral geniculate; GE, ganglionic eminence; Hi, hippocampus; HTh, hypothalamus; Th, thalamus. Scale bars: $A, B, D, 200 \mu m ; C, H-J, 500$ $\mu \mathrm{m} ; \boldsymbol{E}-\boldsymbol{G}, 1 \mathrm{~mm}$

were on C57BL/6J background. All embryos examined in this study were collected regardless of sex.

Histology and DiI tracing. Established in situ hybridization and immunohistochemical procedures with previously characterized probes and antibodies were used (Stumm et al., 2002; Sánchez-Alcañiz et al., 2011; Suzuki-Hirano et al., 2011). A Sox2 probe (GI:127140985, nucleotides 418-1681) was generated by RT-PCR cloning. Primary antibodies are listed in Table 1. Axon tracing was performed as described previously (Molnár et al., 1998).

Thalamic explant cultures. Thalami were prepared from E13.5 Cxcr4$\mathrm{GFP}^{+}$brains and cut into $300 \times 300 \mu \mathrm{m}^{2}$ pieces using a McIlwain tissue chopper. Explants were placed onto collagen-coated coverslips, embedded in collagen ( $3 \mathrm{mg} / \mathrm{ml}$, BD Biosciences) and cultured in Neurobasal Medium (Thermo Fisher Scientific) supplemented with 2 mm glutamine, 5\% FBS, and $100 \mathrm{U} / \mathrm{ml}$ Pen-Strep (all PAA). CXCL12 (Peprotech) and AMD3100 (Sigma-Aldrich) were added immediately after plating. Native GFP was imaged after $48 \mathrm{~h}$ in fixed explants.

Image acquisition and analysis. Confocal images were captured with an LSM510 Meta; other micrographs were taken using an Axio Imager A1 (Zeiss). Throughout the study, $\mathrm{Cxcl12^{-1- }}$ mice were compared with control littermates $\left(\mathrm{Cxcl1}^{+/+}\right.$or $\left.\mathrm{Cxcl12}^{+/-}\right)$. All measurements (thalamus area, intracortical TCA length, and cell counts) were normalized to mean values of litter controls. The area of the developing thalamus was determined based on Gbx2, Sox2, and Slc17a6 patterns using ImageJ. Intracortical TCA length was determined using ImageJ as illustrated in Results (see Fig. 6F). Quantification of interneuron distribution in the cortex has been described (Abe et al., 2014). Axon outgrowth from thalamus explants was quantified by measuring the 20 longest axons in each explant with ImageJ using the NeuronJ plug-in (illustrated in Fig. 6G). In each experimental run, axon length was normalized to control mean of the respective run. Statistics were calculated and plotted with GraphPad Prism and IBM SPSS software. Adobe Photoshop and InDesign CS6 were used to process and arrange figures.

\section{Results}

Meninges are the primary CXCL12 source for early CXCR4expressing thalamic neurons

Little is known about the expression of the Cxcl12, Cxcr4, and $\mathrm{Cxcr} 7$ genes in the developing thalamus. We thus analyzed spatiotemporal expression profiles of these genes by highly sensitive in situ hybridization with radiolabeled probes, focusing on the $G b \times 2^{+}$domain in the developing diencephalon. This domain corresponds to the thalamic mantle zone (tMZ) and is composed of postmitotic glutamatergic neurons, which project to the cortex and constitute the nuclear complex that is traditionally viewed as the thalamus (Chatterjee and Li, 2012). First, we examined E12.5, E13.5, and E14.5 embryos (Fig. 1A-O), because most thalamic neurons are generated during this period (Suzuki-Hirano et al., 2011). Adjacent sections revealed strong expression of Cxcr4 and $\mathrm{Cxcr} 7$ in the progenitor domain along the third ventricle at E12.5 and E.13.5. In contrast, the tMZ expressed CXcr4 but not $C x c r 7$ at this early stage of thalamic development (Fig. $1 A, C, E, F, H, J$ ). At E14.5, the lateral tMZ still exhibited contiguous strong CXcr4 signal and virtually no $C x c r 7$ signal, whereas the medial tMZ contained scattered $\mathrm{Cxcr} 4^{+}$cells and uniform faint $\mathrm{Cxcr} 7$ signal (Fig. $1 \mathrm{~K}, \mathrm{M}, \mathrm{O}$ ). $\mathrm{Cxcl12}$ was expressed in the meninges covering the thalamus but, apart from some forming blood vessels, was not detected within the thalamus during E12.5-E14.5 (Fig. $1 B, G, L$ ). We confirmed localization of Cxcr4 in the thalamus by dual labeling with the thalamic marker SOX2 (Vue et al., 2007). Specifically, dual immunofluorescence for GFP and SOX2 showed that the entire $\mathrm{SOX}_{2}{ }^{+}$domain was $\mathrm{Cxcr} 4-\mathrm{GFP}^{+}$at E14.5 (Fig. $1 P$ $\left.P^{\prime \prime}\right)$. Because of its slow turnover, GFP traces back brain structures that expressed the reporter during the preceding days. Thus, the Cxcr4-GFP pattern at E14.5 corresponds to the finding that vir- 

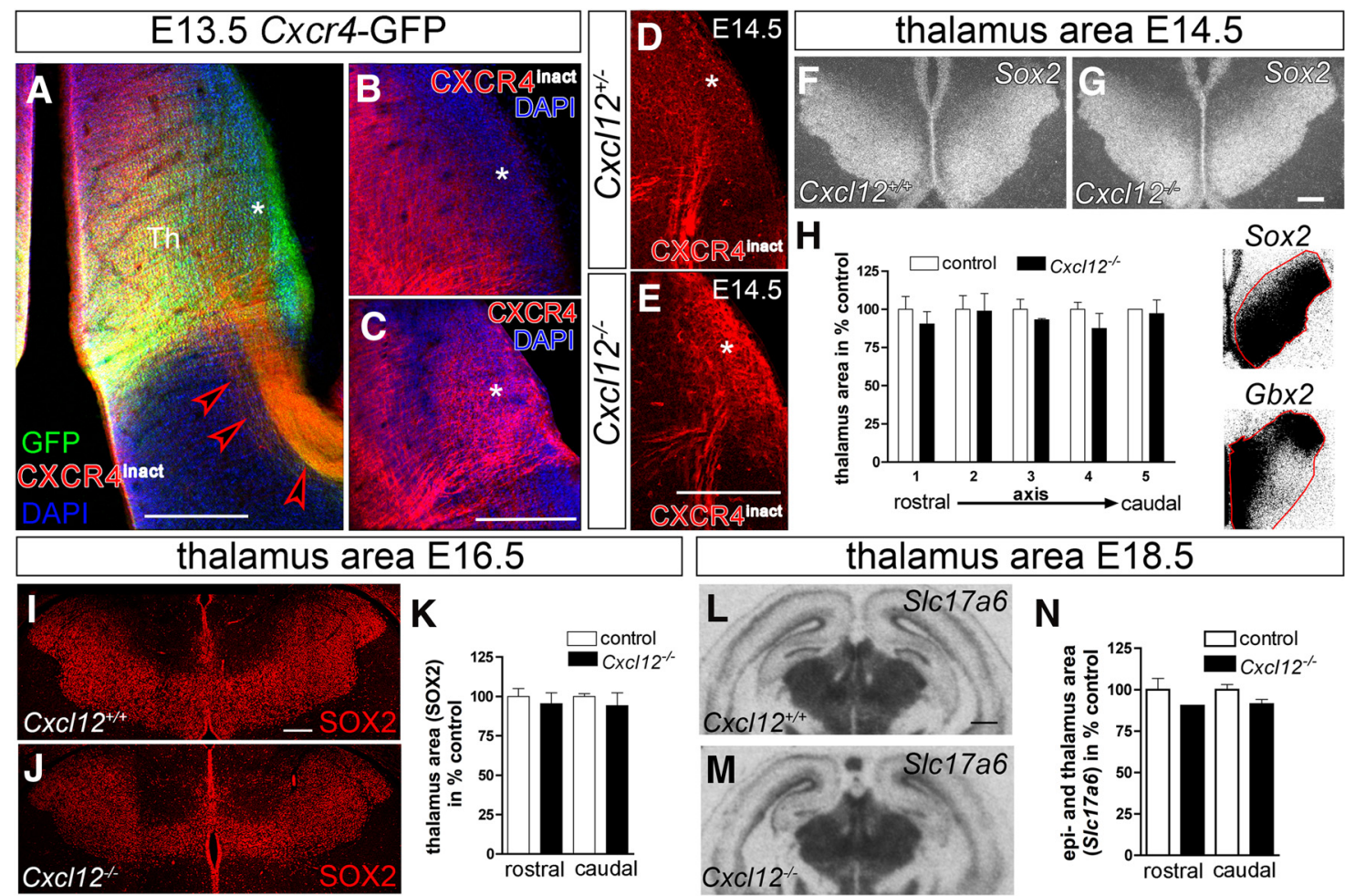

Figure 3. CXCL12 activates CXCR4 receptors in the developing thalamus but is dispensable for thalamus formation. $\boldsymbol{A}-\boldsymbol{E}$, Confocal images show immunohistochemistry for inactive/nonphosphorylated $C X C R 4$ ( $\left.C X C R 4{ }^{\text {inact }} ; A, B, D, E\right)$ and total $C X C R 4$ (CXCR4; $C$ ) in coronal thalamus sections. $A$, Overlay of immunostained GFP, $C X C R 4{ }^{\text {inact }}$, and DAPl in an E13.5 CXCr4-GFP reporter mouse shows CXCR4 in the thalamocortical projection (arrowheads). Note that the lateral thalamus (asterisk) contains only sparse CXCR4 ${ }^{\text {inact }}$ signal despite being $\left(X C Y 4-G F P{ }^{+} . B, C\right.$, The lateral thalamus (asterisk)

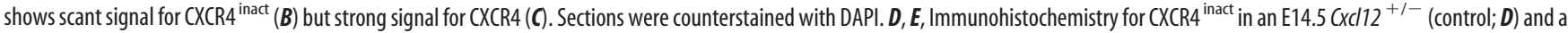
CXCl12 ${ }^{-1-}$ littermate $(\boldsymbol{E})$ demonstrates that XXCR4 ${ }^{\text {inact }}$ increases in the lateral thalamus (asterisks) in the absence of CXCL12. $\boldsymbol{F}, \mathbf{G}$, Dark-field micrographs of emulsion-dipped coronal sections

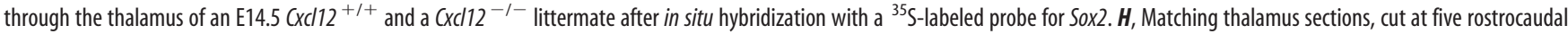
sectional levels in E14.5 control $(n=3)$ and $\left(x c 112^{-1-}\right.$ littermates $(n=3)$, were hybridized for Gbx2 (sectional levels 1, 3, and 5 ) and Sox2 (sectional levels 2 and 4$)$ as thalamus markers. The marker ${ }^{+}$area was determined in micrographs of emulsion-dipped sections after setting a threshold as shown in the insets to the right (signal above threshold appears black). $I, J$, Confocal images

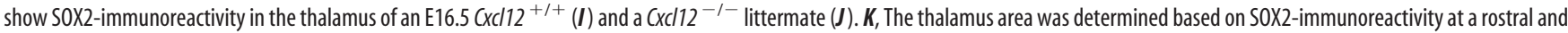
at a caudal sectional level in E16.5 control and $\left(x c / 12^{-1-}\right.$ littermates $(n=5$ each). $\boldsymbol{L}, \boldsymbol{M}, \mathbf{X}$-ray autoradiograms show SIc17a6 (Vglut2) mRNA in hybridized coronal head sections of an E18.5 $\mathrm{CxCl12^{+/+ }}(\boldsymbol{L})$ and a $\mathrm{CxCl12^{-1- }}$ littermate $(\boldsymbol{M}) . \boldsymbol{N}$, The S/C17a6 mRNA-positive area corresponding to thalamus and epithalamus was determined in $x$-ray autoradiograms at a rostral and at a caudal

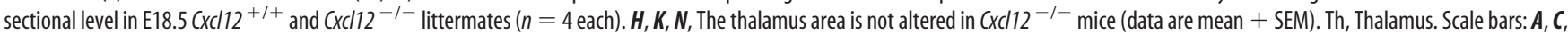
$E, G, I, 200 \mu \mathrm{m} ; L, 500 \mu \mathrm{m}$.

tually the entire forming thalamus expresses Cxcr4 mRNA at E12.5 and E13.5. Dual in situ hybridization showed that at E14.5 Cxcr4 mRNA was still expressed in Sox $2^{+}$and $G b \times 2^{+}$parts of the thalamus (Fig. 2A-D).

Next, we examined stages during which thalamic nuclei begin to differentiate (E16.5, E17.5, P0; demonstrated for E17.5 in Fig. $2 E-P)$. We observed that $C x c r 4$ was progressively downregulated while $\mathrm{Cxcl} 12$ and $\mathrm{Cxcr} 7$ were upregulated in the thalamus. Moderate to faint contiguous Cxcr4 signal could be detected until E17.5 in dorsolateral nuclei. Scattered, strongly Cxcr $4^{+}$cells were present throughout the thalamus at all stages.

Our gene expression analyses suggest that CXCR4 is the predominantly expressed CXCL12 receptor in early postmitotic thalamocortical neurons and that the meninges are the primary diencephalic CXCL12 source. To test this assumption, we studied CXCR4 receptor activation, making use of UMB-2 and 2B11 anti-CXCR4 antibodies. UMB-2 recognizes its epitope within the amino acid residues 343-352 only when the serine cluster S346-8 is unphosphorylated. As this cluster becomes immediately phosphorylated upon CXCL12 stimulation (Mueller et al., 2013), UMB-2 binds only inactive CXCR4 receptors (Fig. $3 A, B, D, E$; CXCR $\left.4^{\text {inact }}\right) .2 \mathrm{~B} 11$ is directed against the CXCR $4 \mathrm{~N}$ terminus and recognizes CXCR4 receptors independently of their activation state (Fig. 3C; CXCR4). By applying these antibodies to adjacent sections from E13.5 Cxcr4-GFP animals, we found that 2B11 and UMB-2 generated staining medially in the Cxcr4-GFP ${ }^{+}$thalamus, whereas only 2B11 produced staining laterally in the Cxcr4$\mathrm{GFP}^{+}$thalamus (Fig. $3 A-C$; asterisks identify the lateral thalamus). To prove that lack in UMB-2 signal reflects CXCR4 activation in the lateral thalamus, we compared thalamic UMB-2 staining in E14.5 Cxcl12 $2^{-1-}$ and control littermates. In the absence of CXCL12, there was a profound increase in UMB-2 signal in the lateral thalamus (Fig. $3 D, E$, asterisks). These findings indicate active signaling of the CXCL12/CXCR4 pathway in early thalamic neurons.

Development of the thalamus in $\mathrm{Cxcl12^{-1- }}$ mice

We then asked whether CXCL12 is required for thalamus formation. We thus examined thalamic size and shape in $\mathrm{Cxcl12^{-/- }}$ mice at different developmental stages. At E14.5, we alternately hybridized five sections at different rostrocaudal planes for $G b \times 2$ and Sox 2 to identify the boundaries between thalamus and epithalamus (Gbx2) and thalamus and prethalamus (Sox2; Vue et al., 2007). The thalamic area was defined as shown in Figure $3 H$, insets. We found that size and shape of the Gbx2 and Sox2 expression domains were not altered in $\mathrm{Cxcl}_{12} 2^{-1-}$ mice (Fig. $3 \mathrm{~F}-\mathrm{H}$ ). 


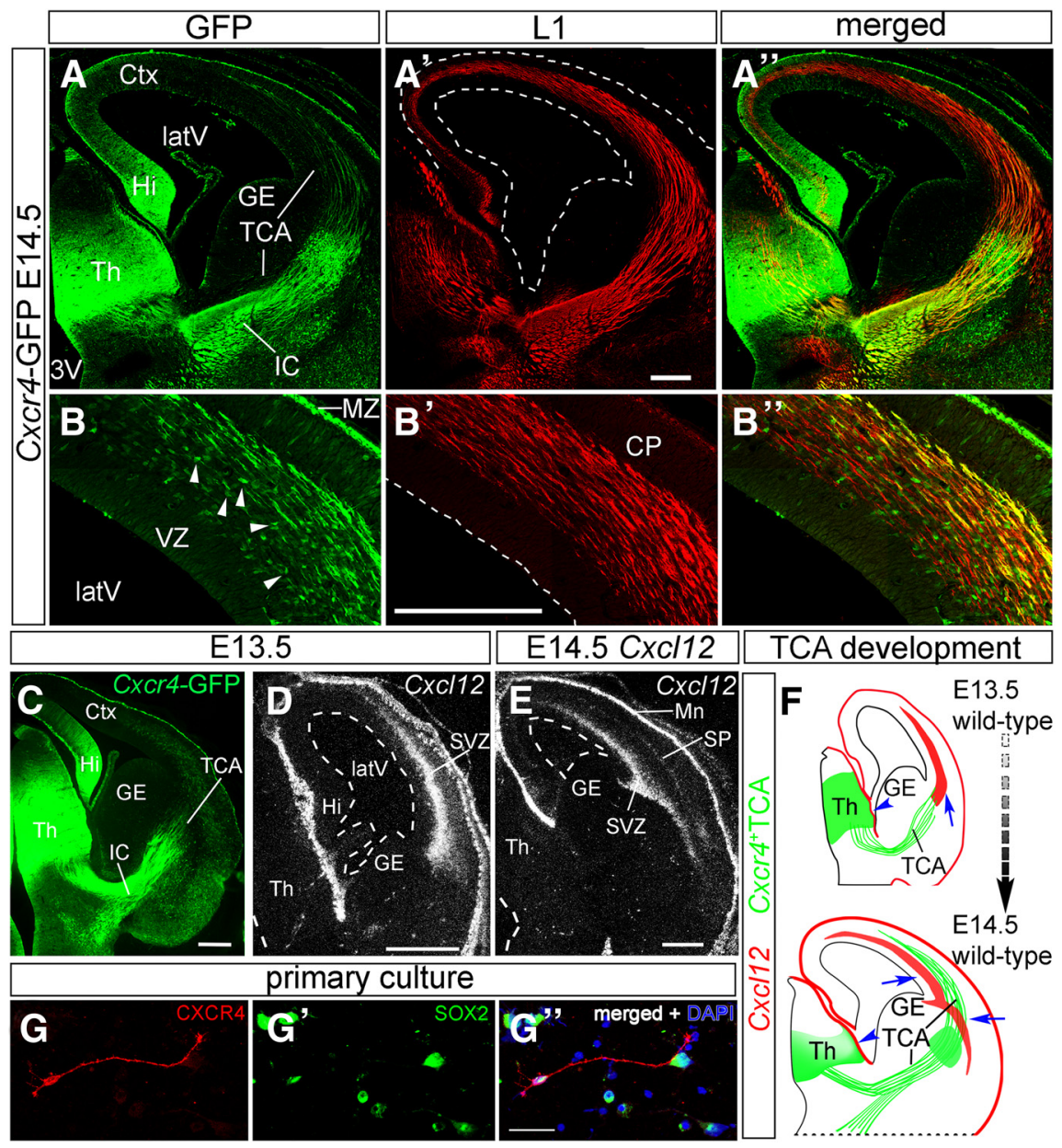

Figure 4. CXCr4-GFP identifies TCAs. $\boldsymbol{A}-\boldsymbol{B}^{\prime \prime}$, Confocal images show immunostained GFP and the axonal marker L1 in an E14.5 CXcr4-GFP reporter mouse. CXcr4-GFP labels TCAs identified by L1. Presumptive GFP ${ }^{+}$interneurons (B, arrowheads) are L1negative. C, Confocal image of a $X$ Cr4-GFP reporter mouse shows that GFP ${ }^{+}$TCAs have traversed the internal capsule (IC) and reached the cortical SVZ at E13.5. D, E, Dark-field micrographs of emulsion-dipped coronal sections at E13.5 (D) and E14.5 (E) after in situ hybridization with a ${ }^{35}$ S-labeled probe for $C x / 12$. CXCl12 is expressed in the cortical SVZ, subplate (SP) and meninges (Mn) covering thalamus and cortex. $\boldsymbol{F}$, Schematic summarizing patterns of Xxr4-GFP and CxCl12 mRNA at E13.5 and E14.5. Arrows indicate putative cortical and arrowheads putative meningeal stimulation sites of $\mathrm{CXCr}^{+}{ }^{+} \mathrm{TCAs}$ and thalamic neurons, respectively. $G$, Dissociated thalamic neurons were immunostained for SOX2 and CXCR4 and counterstained with DAPI. Note CXCR4 in the neurite and the growth-cone. 3V, Third ventricle; $\mathrm{Ctx}$, cortex; GE, ganglionic eminence; Hi, hippocampal anlage; latV, lateral ventricle; Th, thalamus; $V Z$, ventricular zone. Scale bars: $\boldsymbol{A}^{\prime}, \boldsymbol{B}^{\prime}, \mathbf{C}-\boldsymbol{E}, 200 \mu \mathrm{m} ; \boldsymbol{G}^{\prime \prime} 20 \mu \mathrm{m}$.

The thalamic boundaries were sharply delineated in the mutants and appeared similar to controls (shown for Sox2 in Fig. $3 F, G$ ). We then immunostained for SOX2 at E16.5 (Fig. 3I-K) and hybridized for vesicular glutamate transporter 2 (Vglut2/Slc17a6; Fig. $3 L-N)$ at E18.5. Again, we found the labeled areas to be of normal size and shape in $\mathrm{Cxcl1} 2^{-1-}$ embryos, suggesting that the thalamus forms independently of CXCL12. However, because Gbx2, Sox2, and Vglut2 do not resolve thalamic subnuclei, these findings do not preclude the possibility that genesis of distinct thalamic nuclei might require CXCL12.

\section{CXCR4 ${ }^{+}$TCAs traverse a Cxcl12-expressing domain in the cortical SVZ}

Having identified CXCR4 in neurons forming thalamocortical projections, we wondered whether CXCR4 is functionally involved in TCA development. To address this question, we used the Cxcr4-GFP reporter for visualization of TCAs emerging from Cxcr4-expressing neurons. Coimmunostaining for GFP and the axonal marker $\mathrm{L} 1$ revealed $\mathrm{Cxcr} 4-\mathrm{GFP} / \mathrm{L} 1^{+}$axons originating in the thalamus and extending through the internal capsule toward the cortex (Fig. $\left.4 A-B^{\prime \prime}\right)$. GFP patterns in the E14.5 cortex (Fig. $4 B-B^{\prime \prime}$ ) were consistent with presence of Cxcr4-GFP in axons, interneurons, and Cajal-Retzius cells, and absence of Cxcr4-GFP from cortical PNs (Stumm et al., 2003). These findings establish that Cxcr4-GFP specifically labels TCAs and rule out the possibility that corticothalamic axons are labeled.

Next, we examined where along the TCA trajectory Cxcl12 is expressed during E12.5-E14.5 by comparing patterns of Cxcr4-GFP and Cxcl12 mRNA. Areas in the ventral thalamus, prethalamus, internal capsule, and ventral telencephalon were traversed by Cxcr4-GFP ${ }^{+}$TCAs before E13.5 but exhibited only very sparse Cxcl12 signal (shown for E13.5 in Fig. $4 C, D)$. By E13.5, Cxcr4-GFP ${ }^{+}$TCAs reached the pallial-subpallial boundary and started to enter the Cxcl12-expressing domain in the cortical SVZ (Fig. 4C, D, F). Between E13.5 and E14.5, Cxcr4-GFP ${ }^{+}$ TCAs crossed the Cxcl12-expressing SVZ and progressed considerably within the cortex, covering more than one-half of its lateromedial extent (Fig. 4A-F). TCA growth dynamics observed with Cxcr4GFP are consistent with findings by DiI tracing (Auladell et al., 2000).

A prerequisite for a direct influence of CXCL12 on TCAs is the presence of CXCR4 receptors in the axonal compartment. We observed prominent CXCR4 immunolabeling in Cxcr4-GFP ${ }^{+}$TCAs entering the internal capsule (Fig. $3 A$, arrowheads). The receptor could also be identified in the distal axonal compartment (data not shown) but the signal was less intense than proximally, where TCAs are bundled. To confirm that CXCR4 is targeted toward the growth cone of neurites extending from thalamic neurons, we immunostained CXCR4 and SOX2 in thalamic cultures. This revealed CXCR4 presence at the soma, neurite, and growth cone of SOX $2^{+}$thalamic neurons (Fig. $4 G-G^{\prime \prime}$ ). Together, these findings prompt to suggest that the thalamic meninges and the cortical SVZ are CXCL12 sources that influence TCA development through somatic and axonal CXCR4 stimulation, respectively.

\section{CXCL12 promotes intracortical TCA elongation}

To directly test whether CXCL12 is involved in TCA development, we traced the trajectories by DiI placement in control and $\mathrm{Cxcl12}^{-/-}$E14.5 and E16.5 thalami (Fig. 5A-H). No misrouting of DiI-labeled TCAs was observed in the ventral thalamus, internal capsule, and ventral telencephalon of $\mathrm{Cxcl1}^{-/-}$mice. However, labeled axons appeared less developed in the cortex of these mutants. Measurement of the intracortical length of DiI-labeled TCAs confirmed significantly delayed TCA growth in $\mathrm{Cxcl1} 2^{-/-}$ embryos compared with control littermates (E14.5, $p=0.0003$ and E16.5, $p=0.009$ for genotype, two-way ANOVA; Fig. $5 I, J)$. 


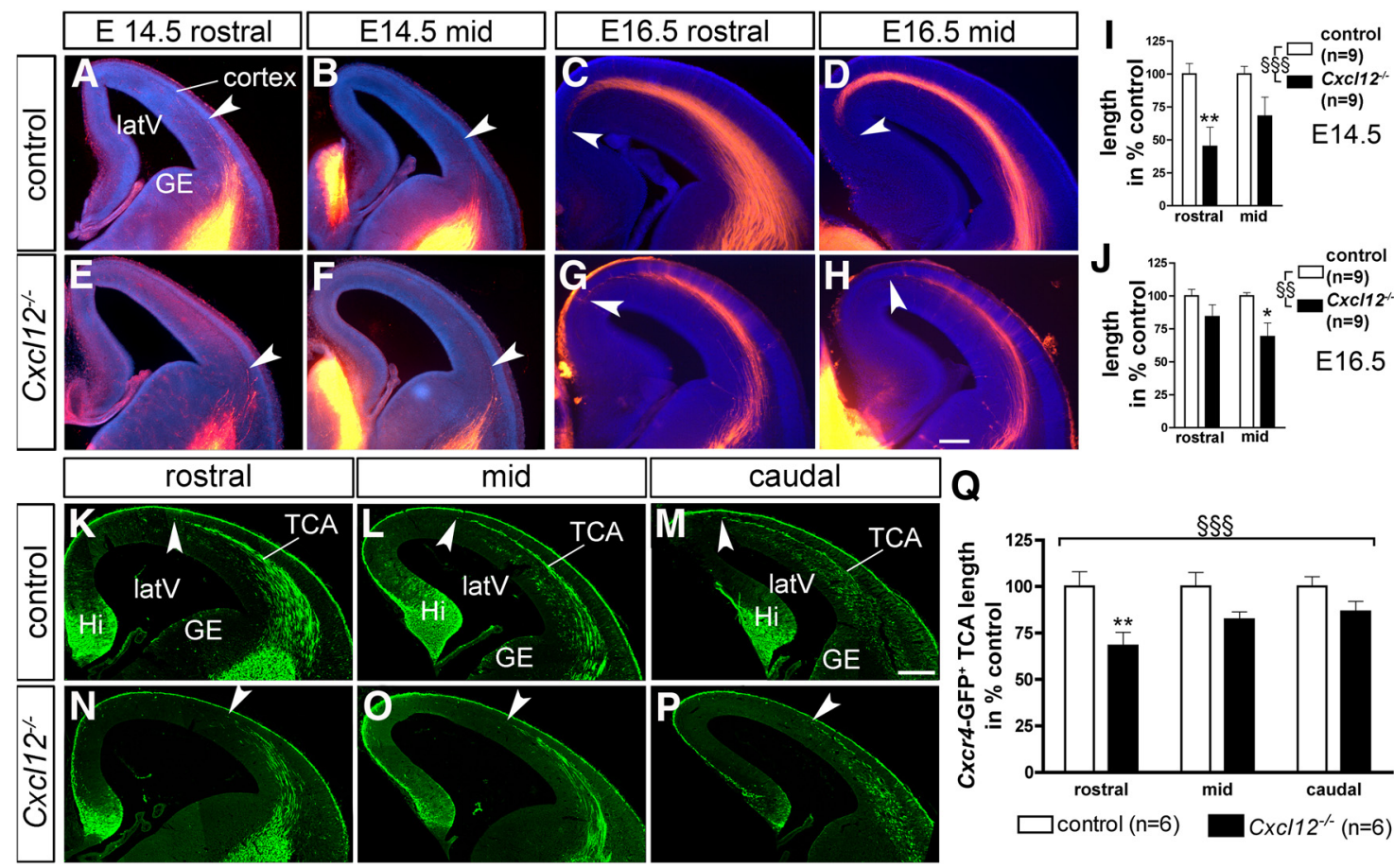

Figure 5. Deletion of $C x C / 12$ attenuates intracortical TCA growth. $\boldsymbol{A}-\boldsymbol{H}$, Thalamic Dil labeling at $\mathrm{E} 14.5(\boldsymbol{A}, \boldsymbol{B}, \boldsymbol{E}, \boldsymbol{F})$ and $\mathrm{E} 16.5(\boldsymbol{C}, \boldsymbol{D}, \boldsymbol{G}, \boldsymbol{H})$ shown in coronal sections at a rostral and a mid-sectional level in control $(\boldsymbol{A}-\boldsymbol{D})$ and $\left(X \mathrm{C} / 12^{-1-}\right.$ embryos $(\boldsymbol{E}-\boldsymbol{H})$. Arrowheads point to positions of the most advanced Dil-labeled TCAs. $\boldsymbol{I}, \boldsymbol{J}$, Mean and SEM of relative intracortical length of Dil-labeled TCAs in E14.5 $(\boldsymbol{I})$ and E16.5 $(\boldsymbol{J})$ control embryos and $\left(x \mathrm{C} / 12^{-/-}\right.$littermates at a rostral and mid-sectional level. $\boldsymbol{K}-\boldsymbol{P}$, Confocal images show immunostained GFP in control $(\boldsymbol{K}-\boldsymbol{M})$ and $\left(\mathbf{X} \mathrm{C} / 12^{-/-}(\boldsymbol{N}-\boldsymbol{P})\right.$ E14.5 Cxcr4-GFP reporter mice at rostral $(\boldsymbol{K}, \boldsymbol{N})$, mid $(\boldsymbol{L}, \mathbf{0})$, and caudal sectional planes $(\boldsymbol{M}, \boldsymbol{P})$. Arrowheads point to positions of the most advanced GFP ${ }^{+}$TCAs. $\boldsymbol{Q}$, Mean and SEM of relative intracortical length of $\mathrm{CXCr4-GFP}{ }^{+}$TCAs in control and Cxcl12 ${ }^{-/-}$E14.5 CXcr4-GFP reporter mice at rostral, mid, and caudal sectional planes. Statistics in I, J, Q: ${ }^{\$ \S \S} p<0.001,{ }^{\S \S} p<0.01$ for genotype (two-way ANOVA). ${ }^{* *} p<0.01,{ }^{*} p<0.05$ (Bonferroni's post hoc test). Numbers of analyzed embryos are indicated. GE, Ganglionic eminence; Hi, hippocampal anlage; latV, lateral ventricle. Scale bars: $\boldsymbol{H}, \boldsymbol{M}, 200 \mu \mathrm{m}$.

Length reductions by $55.0 \%$ at the rostral sectional level at E14.5 $(p<0.01)$ and by $31.0 \%$ at the mid-sectional level at E16.5 $(p<$ $0.05)$ were significant in the post hoc test.

To focus specifically on Cxcr4 ${ }^{+}$TCAs, we crossed Cxcr4-GFP into the $\mathrm{Cxcl12^{+/- }}$ mouse line and generated Cxcr4-GFP; Cxcl12 ${ }^{-1-}$ mutant embryos and Cxcr4-GFP control littermates (Fig. $5 \mathrm{~K}-\mathrm{P}$ ). Length measurement of $\mathrm{GFP}^{+}$fibers in the E14.5 cortex at three rostrocaudal sectional levels showed an overall growth reduction in the mutants compared with control littermates ( $p=0.0004$ for genotype, two-way ANOVA; Fig. 5Q). Length reduction by $31.8 \%$ at the rostral sectional level reached significance in the post hoc test $(p<0.01)$. The delayed growth was not accompanied by misrouting of Cxcr4-GFP ${ }^{+}$TCAs in Cxcr4-GFP;Cxcl12-I- embryos.

Next, we tested whether CXCL12 overexpression accelerates TCA development. We thus generated double-transgenic mice expressing Cxcr4-GFP and a CXCL12-RFP fusion protein under control of the Cxcl12 promoter (CXCL12-RFP). In CXCL12-RFP mice, additional $\mathrm{Cxcl12}$ gene copies result in increased $\mathrm{Cxcl12}$ mRNA expression (Fig. $6 A, B$ ) and, hence, in an increase of functional CXCL12. Immunofluorescent colabeling of Cxcr4-GFP and neurofilament (NF) as a TCA marker in E15.5 Cxcr4-GFP control and CXCL12-RFP;CXcr4-GFP double-transgenic mice revealed accelerated TCA growth following CXCL12 overexpression (Fig. 6C,D). Remarkably, in an area corresponding to the visual cortex of CXCL12-RFP embryos, TCAs entered the CP prematurely and even targeted the MZ (Fig. $6 D$, asterisks). Measurement of intracortical TCA length at seven rostrocaudal sectional planes confirmed increased TCA length in CXCL12-RFP animals ( $p=0.0001$ for genotype, two-way ANOVA; Fig. $6 E$ ). An increase in intracortical TCA length by $28 \%$ in the second sectional plane was significant in the post hoc test $(p<0.05)$. These gain and loss of function experiments establish that CXCL12 promotes intracortical progression of TCAs.

\section{CXCR4 mediates a growth-promoting effect of CXCL12 on TCAs}

Defective CXCL12/CXCR4 signaling affects the development of cortical cell populations including interneurons and CajalRetzius cells (Stumm and Hollt, 2007). Thus, impaired intracortical TCA growth in $\mathrm{Cxcl12^{-/- }}$ mice may be caused indirectly by defects in cortical development or reflect absence of growthpromoting CXCR4 signals in TCAs. To discriminate between these possibilities, we examined axon outgrowth in E13.5 Cxcr4$\mathrm{GFP}^{+}$thalamic explant cultures (Fig. $6 G-K$ ). Stimulation with CXCL12 dose-dependently increased outgrowth of Cxcr4-GFP ${ }^{+}$ axons $(p<0.0001$, ANOVA; Fig. $6 L)$. Outgrowth was increased by $15.9 \%$ after $20 \mathrm{nM}(p>0.05)$ and by $33.7 \%$ after $40 \mathrm{nM}$ CXCL12 $(p<0.001)$ versus the nonstimulated control. The CXCR4 antagonist AMD3100, added at $6 \mu \mathrm{M}$, blocked the growth-promoting effect of $40 \mathrm{nM}$ CXCL12 $(p<0.01)$ but had no significant effect when applied alone. These findings suggest that a CXCL12/CXCR4 pathway exerts a direct growth-promoting effect on TCAs.

IPC-derived CXCL12 ensures efficient TCA growth and interneuron dispersion

We then asked, whether SVZ-derived CXCL12 is sufficient to promote TCA growth. To address this, we deleted Cxcl12 selectively in IPCs using $\mathrm{Tbr}_{2} \mathrm{Cre} /+^{+}$-mediated excision of the CXCL12- 

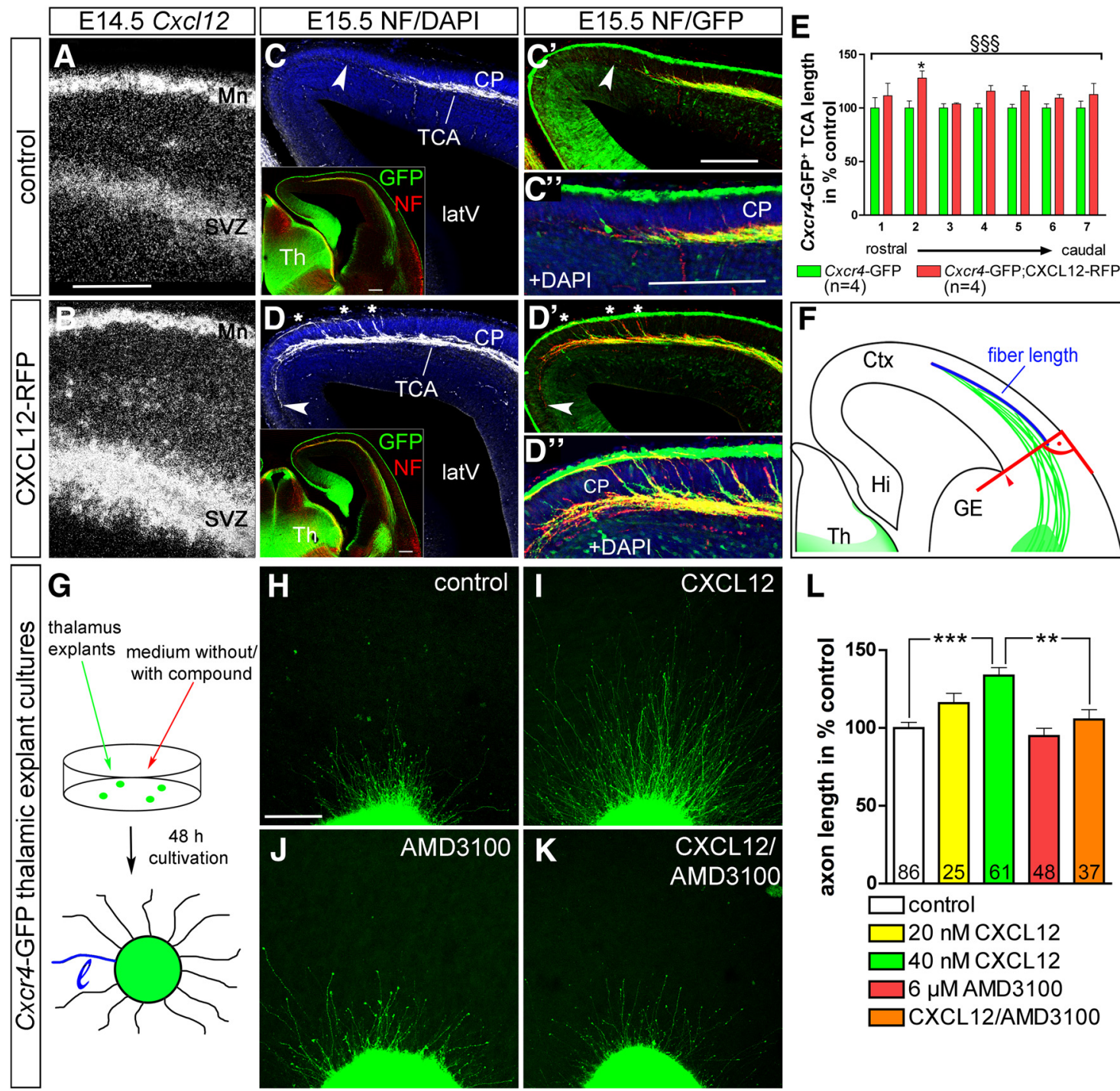

Figure 6. A CXCL12/CXCR4 pathway accelerates intracortical TCA growth. $A, B$, Dark-field views of the cerebral cortex at E14.5 after in situ hybridization with a ${ }^{35}$ S-labeled probe for $C X C 112$.

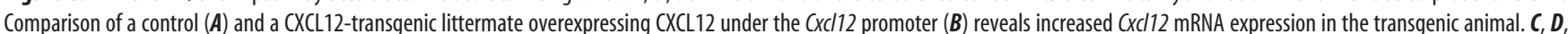

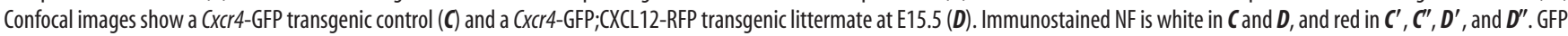
is green in $\boldsymbol{C}^{\prime}, \boldsymbol{C}^{\prime \prime}, \boldsymbol{D}^{\prime}$, and $\boldsymbol{D}^{\prime \prime}$. DAPI is shown in blue in $\boldsymbol{C}^{\prime} \mathbf{D}, \boldsymbol{C}^{\prime \prime}$, and $\boldsymbol{D}^{\prime \prime}$. Arrowheads point to the location of the most advanced stained TCAs; asterisks point to TCAs crossing the $C P$ prematurely $(\boldsymbol{D}$, $\left.D^{\prime}\right)$. E, Mean and SEM of relative intracortical length of $C X C r 4-G F P{ }^{+}$TCAs in CXCr4-GFP and CXCr4-GFP;CXCL12-RFP E15.5 mice in seven sectional planes along the rostrocaudal axis. $F$, Scheme illustrating measurement of intracortical TCA length. G, Scheme illustrating experimental procedure of explant culturing. Thalamus explants were prepared from E13.5 (xCr4-GFP mice and cultured for $2 \mathrm{~d}$ in medium supplemented with vehicle (control), $20 \mathrm{~nm}$ CXCL12, $40 \mathrm{~nm}$ CXCL12, or $6 \mu \mathrm{m}$ CXCR4 antagonist AMD3100. In each explant, length (I) of the 20 longest axons was measured, normalized to control and expressed as mean + SEM. $\boldsymbol{H}-\boldsymbol{K}$, Representative $\left(X C r 4-G F{ }^{+}\right.$thalamus explants receiving vehicle $(\boldsymbol{H}), 40 \mathrm{~nm}$ CXCL12 (I), AMD3100 (J), and $40 \mathrm{~nm}$ CXCL12 + AMD3100 (K). $L, C X C L 12$ dose-dependently stimulates axonal growth in thalamus explants (for each group, the number of analyzed explants is given in the corresponding bar). Statistics, $E:{ }^{\$ \$ \S} p<0.001 \mathrm{for}$ genotype (two-way ANOVA), ${ }^{*} p<0.05$ (Bonferroni's post hoc test); $L:{ }^{* *} p<0.001,{ }^{* *} p<0.01$ (one-way ANOVA and Tukey's post hoc test). Data are mean + SEM. Ctx, Cortex; GE, ganglionic eminence; Hi, hippocampal anlage; latV, lateral ventricle; Mn, meninges; Th, thalamus. Scale bars: $A, C$ (inset), $\boldsymbol{C}^{\prime}, \boldsymbol{C}^{\prime}, \boldsymbol{D}$ (inset), $\boldsymbol{H}, 200 \mu \mathrm{m}$.

encoding exon2 in Cxcl12 ${ }^{\mathrm{LoxP} /-}$ mice $\left(\mathrm{Cxcl} 12 \mathrm{cKO}{ }^{\mathrm{Tbr} 2-\mathrm{Cre}}\right.$; Costello et al., 2011; Tzeng et al., 2011). In situ hybridization with an exon2-selective probe confirmed that Cxcl12 expression was indeed disrupted in the SVZ and intact in the meninges in E14.5 Cxcl12cKO ${ }^{\text {Tbr2-Cre }}$ mice (Fig. $7 A, B$ ). To visualize Cxcr4expressing TCAs in these mutants, we generated E14.5 Cxcr4GFP;Cxcl12cKO ${ }^{\text {Tbr2-Cre }}$ mice by crossbreeding $\mathrm{Tbr} 2^{\mathrm{Cre}}$ driver, Cxcr4-GFP reporter and Cxcl1 $2^{\mathrm{LoxP} /-}$ mice (Fig. $7 C, D$ ). Intracortical length of Cxcr4-GFP ${ }^{+}$TCAs, determined at three rostrocaudal sectional levels, was reduced in the conditional mutants compared with littermate controls $(p<0.0001$ for genotype, two-way ANOVA; Fig. 7E). The post hoc test identified that TCAs were significantly shorter by 28,21 , and $25 \%$ at the rostral $(p<$
$0.001)$, medial $(p<0.05)$, and caudal $(p<0.01)$ level in the conditional mutants.

Having shown that IPC-derived CXCL12 promotes elongation of TCAs in the SVZ, we wondered whether IPCs have a similar effect on interneurons. It is well established that the CXCL12/CXCR4/CXCR7 signaling module regulates interneuron migration in the cortex, but the respective contributions of meningeal- and IPC-derived CXCL12 to this process has not been dissected by targeting the Cxcl12 gene selectively in one of these tissues. We thus examined interneuron positioning in Cxcl12cKO ${ }^{\mathrm{Tbr2}-\mathrm{Cre}}$ mice. As $\mathrm{GFP}^{+}$TCAs mask the position of weakly GFP-stained interneurons in the cortex of Cxcr4-GFP mice, we hybridized for $L h x 6$, a marker of medial ganglionic 

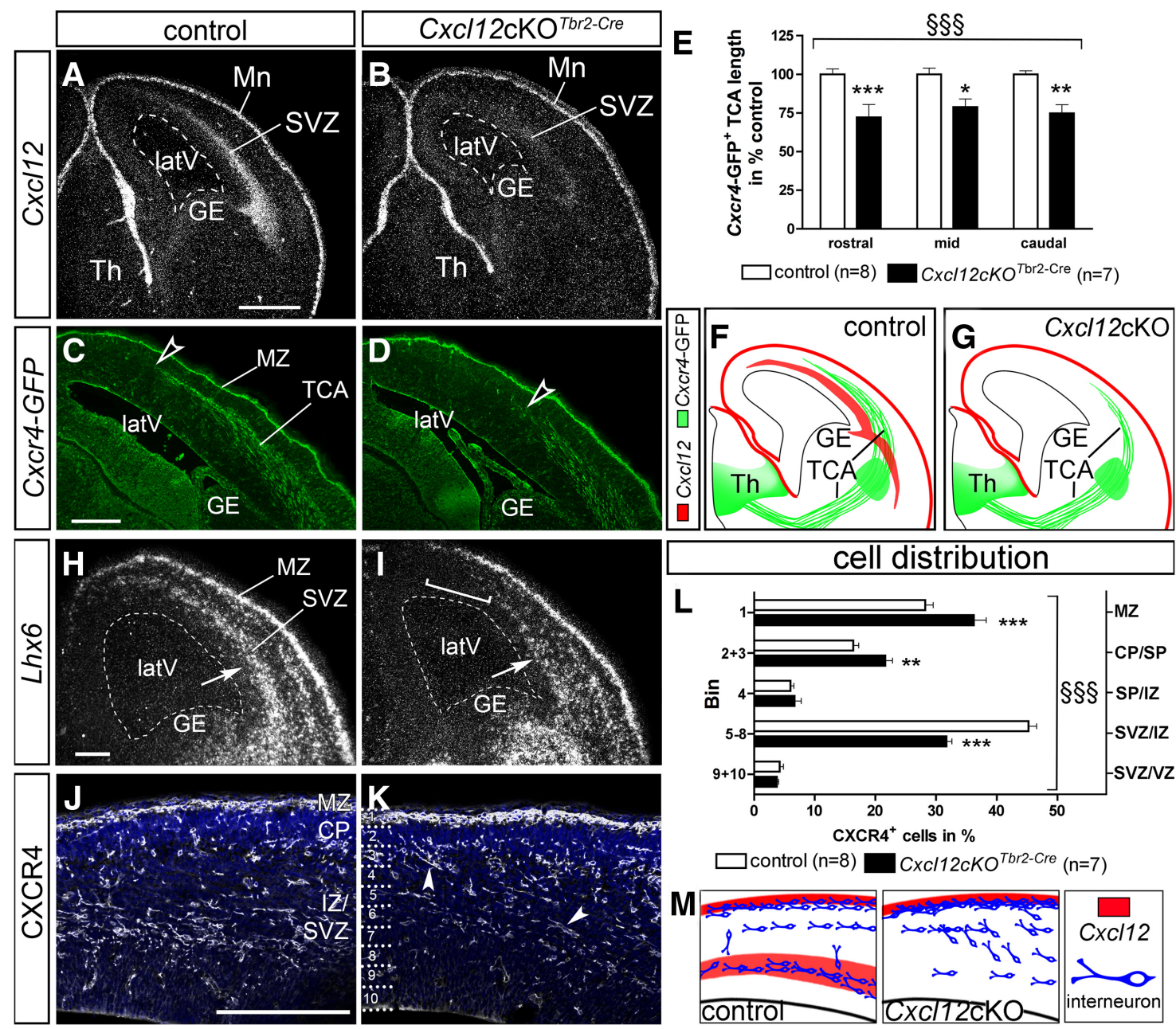

Figure 7. IPC-derived CXCL12 ensures efficient intracortical TCA growth and interneuron dispersion. $A, B$, Dark-field micrographs of emulsion-dipped E14.5 coronal brain sections after in situ

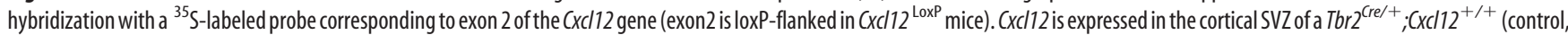

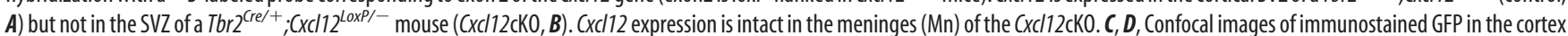
at mid-rostrocaudal sectional level in E14.5 Cxc112cKO and control mice carrying the CXCr4-GFP reporter. Arrowheads pointing to the most advanced GFP ${ }^{+}$TCAs demonstrate that GFP $^{+}$TCAs are

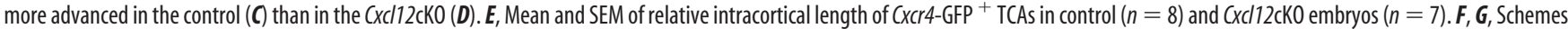
illustrating the TCA phenotype in the $C X C 172 \mathrm{KO}$. $\boldsymbol{H}, \boldsymbol{I}$, Dark-field micrographs of emulsion-dipped coronal sections through the E14.5 cortex after in situ hybridization with a ${ }^{35} \mathrm{~S}$-labeled probe for

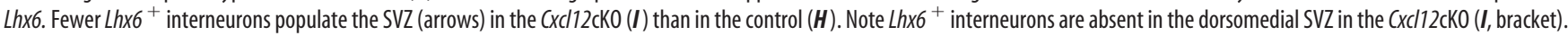
$\boldsymbol{J}, \boldsymbol{K}$, Confocal images of immunostained XCR4 (white) and DAPI (blue) in coronal cortical sections of E14.5 control $(\boldsymbol{J})$ and $\left(X C / 12 c K 0\right.$ mice $(\boldsymbol{K})$. Cortical bins are shown in $\boldsymbol{K}$. There are fewer CXCR4 ${ }^{+}$ interneurons in the SVZ (bins 5-8) and more CXCR4 ${ }^{+}$interneurons in upper cortical layers (bins 1-3) of the CXC112CKO $(K)$ than in the corresponding areas of the control $(J)$. More $C X C R 4{ }^{+}$ interneurons exhibit oblique orientation in the $X X / 12 \mathrm{cKO}$ ( $K$, arrowheads) than in the control, indicating abnormal, surface-directed migration of these cells. $L$, Distribution of $C X C R 4{ }^{+}$cells in the cortex of E14.5 control $(n=8)$ and CXI12cKO mice $(n=7)$. Data are mean + SEM of CXCR4 ${ }^{+}$cells per area in percentage of all CXCR4 ${ }^{+}$cells. $\boldsymbol{M}_{\text {, Scheme illustrating the }}$ interneuron phenotype in the $C x \mathrm{C} / 12 \mathrm{CKO}$. Statistics: ${ }^{\S \S} p<0.0001$ for genotype $(\boldsymbol{E})$ and bin/genotype interaction ( $\boldsymbol{L}$; two-way ANOVA). ${ }^{* * *} p<0.001,{ }^{* *} p<0.01,{ }^{*} p<0.05$ (Bonferroni's post hoc test compared with control); $n$ numbers as indicated. GE, ganglionic eminence; latV, lateral ventricle; Mn, meninges; SP, subplate; Th, thalamus; VZ, ventricular zone. Scale bars: $A, 500 \mu \mathrm{m} ; \boldsymbol{C}, \boldsymbol{H}, \boldsymbol{J}, 200 \mu \mathrm{m}$.

eminence-derived interneurons. In the two examined E14.5 Cxcl12cKO ${ }^{\text {Tbr2-Cre }}$ mice, the lateral SVZ contained fewer $\operatorname{Lh} x 6^{+}$ cells than the corresponding region in control littermates (Fig. $7 \mathrm{H}, \mathrm{I}$, arrows). The medial SVZ of the conditional mutants was devoid of $L h x 6^{+}$cells, whereas it was readily populated by $L h x 6^{+}$ cells in the controls (Fig. 7 H, I, brackets). To confirm these obser-

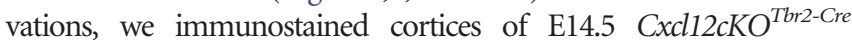
mice for CXCR 4 and counted stained cells in 10 cortical bins in a midcortical area (Fig. $7 J-L$ ). In Cxcl12cKO ${ }^{\text {Tbr2-Cre }}$ mice, there was a significant reduction in CXCR $4^{+}$cells in bins 5-8 corresponding to the SVZ/IZ $(p<0.001)$ and a significant increase in CXCR4 ${ }^{+}$cells in bin $1(p<0.001)$ and bins $2 / 3(p<0.01)$ corresponding to the $\mathrm{MZ}$ and $\mathrm{CP}$, respectively (two-way ANOVA and post hoc test). Leading processes of CXCR4-immunoreactive interneurons migrating in the SVZ/IZ in Cxcl12cKO ${ }^{T b r 2-C r e}$ mice often exhibited an oblique orientation (Fig. $7 K$, arrowheads), suggesting that these cells were heading toward the MZ. In contrast, CXCR4-immunoreactive interneurons migrating in the SVZ/IZ of control mice had a more tangential orientation. In conclusion, CXCL12 produced by IPCs ensures efficient TCA growth and interneuron dispersion in the SVZ/IZ (Fig. $7 F, G, M$ ).

\section{Discussion}

Although considerable progress has been made in identifying molecules that guide migrating cortical interneurons and the forming thalamocortical tract (Hernández-Miranda et al., 2010; Kolodkin and Tessier-Lavigne, 2011; Grant et al., 2012; Price et al., 2012), little is known about mechanisms ensuring that PNs, 


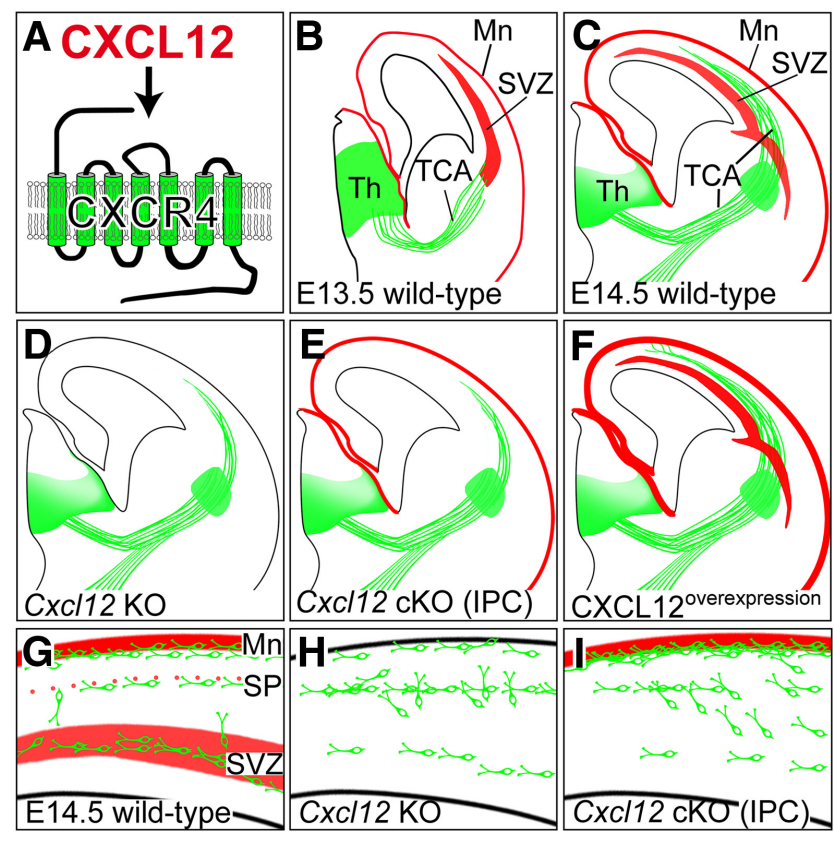

Figure 8. Schematic summarizing $X X C L 12 / C X C R 4$ signaling for TCA and interneuron progression. $A, C x C 172$-expressing structures are shown in red and $C X C r 4$-expressing structures in green. $\boldsymbol{B}, \boldsymbol{C}$, CXCY4-GFP ${ }^{+}$TCAs reach the cortical SVZ at E13.5 and traverse most of the cortex between E13.5 and E14.5. D, E, Intracortical growth of $\mathrm{CXCr} \mathrm{GFP}^{+}{ }^{+}$TCAs is reduced in $\mathrm{CXCl12-}$ deficient mice $(X X / 12$ KO; $D)$ and in conditional mutants lacking CXCL12 in IPCS ( $C X \mid 12$ CKO; $E)$. $F$, Orthotopic CXCL12 overexpression promotes intracortical growth of $\mathrm{CXCr}_{\text {-GFP }}{ }^{+}$TCAs and leads to premature $C P$ invasion and abnormal targeting of the MZ. G, CXCR4-positive interneurons migrate in the MZ, subplate, and SVZ. $H, \operatorname{In} C X C l 12$-deficient mice, interneurons accumulate in the CP/SP area. I, After Cxcl12 ablation in IPCs, interneurons leave the SVZ and seek upper cortical layers. Mn, Meninges; SP, subplate; Th, thalamus.

produced in large numbers in the rapidly growing mammalian cortex, are sufficiently supplied with interneurons and TCAs. A large proportion of cortical PNs arises from IPCs residing in the SVZ (Kolodkin and Tessier-Lavigne, 2011; Vasistha et al., 2014). Intriguingly, the SVZ is traversed by migrating interneurons and ingrowing TCAs during the peak of cortical neurogenesis (Hevner et al., 2004; Wonders and Anderson, 2006; Mire et al., 2012). Findings in the present study are consistent with a role of IPC-derived CXCL12 in promoting intracortical TCA growth through a CXCR4 pathway. To our knowledge, this is the first report to provide evidence that CXCL12 regulates axonal growth within the developing mammalian brain. In addition, we demonstrate that lack of IPC-derived CXCL12 disrupts tangential migration of interneurons in the SVZ/IZ. Our findings imply that while amplifying cortical neurons (Martínez-Cerdeño et al., 2006), IPCs enable efficient progression of TCAs and interneurons by releasing CXCL12 (Fig. 8).

\section{CXCL12 promotes axonal growth in the mammalian brain}

Although CXCL12 effects on axonal growth and axonal guidance have been studied in vitro (Arakawa et al., 2003; Chalasani et al., 2003; Pujol et al., 2005; Heskamp et al., 2013), CXCL12 effects on axonal development within the mammalian brain were not previously resolved. We thus analyzed expression and function of the CXCL12/CXCR4/CXCR7 signaling module in the embryonic thalamus and the forming thalamocortical projection, one of the major intracerebral axonal tracts. We found that CXCR4 receptors are widely present in cell bodies and growing axons of thalamocortical projection neurons. The Cxcr7 gene is tran- scribed in the progenitor domain but not in the differentiation field of the forming thalamus. Examination of the CXCR4 activation state showed that CXCL12, most likely emanating from the meninges covering the thalamus, signals through CXCR4 to the cell bodies of thalamic neurons. Regions in the thalamus, diencephalon, prethalamus, and internal capsule that are traversed by TCAs are largely Cxcl12-negative, suggesting that CXCL12 is not involved in TCA guidance in these regions. Consistently, misrouting of TCAs is not observed in embryos lacking CXCL12. Instead, we present several lines of evidence that CXCL12 stimulates TCA growth. First, CXCL12 enhances axonal growth in thalamus explants through CXCR4. Second, orthotopic CXCL12 overexpression in CXCL12-RFP-transgenic mice leads to increased intracortical TCA growth. Third, intracortical TCA growth is severely attenuated in $\mathrm{Cxcll}^{-/-}$mice. As the thalamic Gbx2 and Sox2 expression domains, which give rise to thalamocortical relay neurons (Chatterjee and Li, 2012), appear normal in $\mathrm{Cxcl1} 2^{-/-}$embryos, it seems unlikely that reduced TCA growth is due to defective thalamus formation. A previous study addressing the role of CXCL12 in axonal development in a mammalian system demonstrated that mesenchymal CXCL12 guides CXCR ${ }^{+}$motor axons once they grow out of the neural tube (Lieberam et al., 2005). Our finding that CXCL12 promotes intracerebral growth of long-ranged axons connecting the thalamus to the late-developing cortex thus unveils a new aspect of CXCL12 function.

\section{Dynamic changes in Cxcr4 expression in the forming thalamus}

Our in situ hybridization results showed that virtually all neurons in the tMZ express Cxcr4 during the early stage of thalamic development (E12.5-E13.5; Fig. 1 $A, F)$. This finding is consistent with a prominent GFP signal observed in the entire tMZ (Fig. $1 P$ ) and most intracortical TCAs (Fig. $6 C^{\prime}, C^{\prime \prime}$ ) in E15.5 Cxcr4-GFP mice, where accumulated GFP protein reflects $C x c r 4$ expression of the preceding days. Also, pioneer axons emerging from the E12.5 thalamus were clearly Cxcr4-GFP ${ }^{+}$. Thus, it is conceivable that the vast majority of TCAs crossing the Cxcl12-expressing SVZ between E13.5 and E14.5 express CXCR4 and may respond to the CXCL12 signal they encounter there. This assumption is supported by our finding that selective ablation of Cxcl12 in the SVZ results in retarded intracortical TCA growth at E14.5.

Between E14.5 and E17.5, Cxcr4 expression ceases in the medial thalamus but is sustained in the lateral thalamus, suggesting that CXCL12 may continuously influence TCAs emerging from lateral nuclei including the dorsal lateral geniculate. In support of this, elevated CXCL12 levels in E15.5 CXCL12-RFP mice cause TCAs to abnormally seek the CP and MZ only in cortical areas that are innervated by lateral thalamic nuclei (Fig. 6D).

It was reported that the growth speed of TCAs is intrinsically regulated by spontaneous activity of thalamic neurons: during the early stage of thalamic development (E12.5-E14.5) high neuronal activity maintains high growth speed, whereas reduced neuronal activity correlates to slower TCA growth during the late stage (E14.5-E16.5; Mire et al., 2012). The E14.5 time point, when TCAs switch from fast to slower growth, corresponds to the time point when Cxcr4 expression decreases in the tMZ. Given that CXCR4 is highly activated in the tMZ between E12.5 and E17.5, a link between CXCR4 signaling, spontaneous activity of thalamic neurons and TCA growth seems worth considering. 


\section{IPC-derived CXCL12 supports tangential migration in the SVZ}

The MZ and SVZ/IZ are major routes of interneurons migrating tangentially in the cortex (Wonders and Anderson, 2006). Cxcl12 mRNA is expressed in the meninges, in the SVZ, and to lesser extent, in the subplate (Stumm et al., 2003, 2007; Abe et al., 2014). The chemokine is attractant for interneurons and supports tangential migration in the cortex. Both CXCL12 receptors, CXCR4 and CXCR7, are involved in this process (Sánchez-Alcañiz et al., 2011; Wang et al., 2011; Abe et al., 2014). The prominent Cxcl12 mRNA signal in the SVZ is localized in IPC (Tiveron et al., 2006). This is a unique constellation because Cxcl12 expression in neuronal progenitors has not been reported in other regions of the embryonic brain. It has been proposed that CXCL12 from IPCs provides a molecular link between IPCs and interneurons (Tiveron et al., 2006; Sessa et al., 2010). This hypothesis was based on the observation that Pax6 and Tbr2 mutants exhibit reduced Cxcl12 expression in the SVZ and abnormal interneuron migration. However, there are severe defects in cortical development in Tbr 2 and Pax 6 mutants and absence of these crucial factors affects expression of a large set of genes other than Cxcl12. It was therefore not clear whether perturbed interneuron migration in $\mathrm{Tbr} 2$ and Pax6 mutants is indeed caused by defective Cxcl12 expression in IPCs (Tiveron et al., 2006; Arnold et al., 2008; Sessa et al., 2010; Georgala et al., 2011; Elsen et al., 2013). We now show that in mice with a selective deletion of Cxcl12 in IPCs, interneurons deviate from their normal migration in the SVZ to seek the remaining meningeal CXCl12 signal, thus providing proof that IPC-derived CXCL12 is essential for interneuron migration in the SVZ.

\section{Conclusion}

Evolution of the cortex is characterized by dramatic expansion of cortical size in mammals. This is enabled by IPC-mediated amplification of cortical neurons. Our study demonstrates that IPC-derived CXCL12 promotes TCA growth and interneuron migration, providing evidence that paracrine signaling mechanisms exist, which coordinate neuronal amplification and invasion of the cortex by extrinsic cell and axon elements.

\section{References}

Abe P, Mueller W, Schütz D, MacKay F, Thelen M, Zhang P, Stumm R (2014) CXCR7 prevents excessive CXCL12-mediated downregulation of CXCR4 in migrating cortical interneurons. Development 141:1857-1863. CrossRef Medline

Arakawa Y, Bito H, Furuyashiki T, Tsuji T, Takemoto-Kimura S, Kimura K, Nozaki K, Hashimoto N, Narumiya S (2003) Control of axon elongation via an SDF-1alpha/Rho/mDia pathway in cultured cerebellar granule neurons. J Cell Biol 161:381-391. CrossRef Medline

Arnold SJ, Huang GJ, Cheung AF, Era T, Nishikawa S, Bikoff EK, Molnár Z, Robertson EJ, Groszer M (2008) The T-box transcription factor Eomes/ Tbr2 regulates neurogenesis in the cortical subventricular zone. Genes Dev 22:2479-2484. CrossRef Medline

Auladell C, Pérez-Sust P, Supèr H, Soriano E (2000) The early development of thalamocortical and corticothalamic projections in the mouse. Anat Embryol 201:169-179. CrossRef Medline

Bhattacharyya BJ, Banisadr G, Jung H, Ren D, Cronshaw DG, Zou Y, Miller RJ (2008) The chemokine stromal cell-derived factor-1 regulates GABAergic inputs to neural progenitors in the postnatal dentate gyrus. J Neurosci 28:6720-6730. CrossRef Medline

Chalasani SH, Sabelko KA, Sunshine MJ, Littman DR, Raper JA (2003) A chemokine, SDF-1, reduces the effectiveness of multiple axonal repellents and is required for normal axon pathfinding. J Neurosci 23:1360-1371. Medline

Chatterjee M, Li JY (2012) Patterning and compartment formation in the diencephalon. Front Neurosci 6:66. CrossRef Medline
Costello I, Pimeisl IM, Dräger S, Bikoff EK, Robertson EJ, Arnold SJ (2011) The T-box transcription factor Eomesodermin acts upstream of Mesp1 to specify cardiac mesoderm during mouse gastrulation. Nat Cell Biol 13: 1084-1091. CrossRef Medline

Elsen GE, Hodge RD, Bedogni F, Daza RA, Nelson BR, Shiba N, Reiner SL, Hevner RF (2013) The protomap is propagated to cortical plate neurons through an Eomes-dependent intermediate map. Proc Natl Acad Sci U S A 110:4081-4086. CrossRef Medline

Georgala PA, Carr CB, Price DJ (2011) The role of Pax6 in forebrain development. Dev Neurobiol 71:690-709. CrossRef Medline

Grant E, Hoerder-Suabedissen A, Molnár Z (2012) Development of the corticothalamic projections. Front Neurosci 6:53. CrossRef Medline

Hernández-Miranda LR, Parnavelas JG, Chiara F (2010) Molecules and mechanisms involved in the generation and migration of cortical interneurons. ASN Neuro 2:e00031. CrossRef Medline

Heskamp A, Leibinger M, Andreadaki A, Gobrecht P, Diekmann H, Fischer D (2013) CXCL12/SDF-1 facilitates optic nerve regeneration. Neurobiol Dis 55:76-86. CrossRef Medline

Hevner RF, Daza RA, Englund C, Kohtz J, Fink A (2004) Postnatal shifts of interneuron position in the neocortex of normal and reeler mice: evidence for inward radial migration. Neuroscience 124:605-618. CrossRef Medline

Kolodkin AL, Tessier-Lavigne M (2011) Mechanisms and molecules of neuronal wiring: a primer. Cold Spring Harb Perspect Biol 3:a001727. CrossRef Medline

Kowalczyk T, Pontious A, Englund C, Daza RA, Bedogni F, Hodge R, Attardo A, Bell C, Huttner WB, Hevner RF (2009) Intermediate neuronal progenitors (basal progenitors) produce pyramidal-projection neurons for all layers of cerebral cortex. Cereb Cortex 19:2439-2450. CrossRef Medline

Li G, Adesnik H, Li J, Long J, Nicoll RA, Rubenstein JL, Pleasure SJ (2008) Regional distribution of cortical interneurons and development of inhibitory tone are regulated by Cxcl12/Cxcr4 signaling. J Neurosci 28:10851098. CrossRef Medline

Lieberam I, Agalliu D, Nagasawa T, Ericson J, Jessell TM (2005) A Cxcl12CXCR4 chemokine signaling pathway defines the initial trajectory of mammalian motor axons. Neuron 47:667-679. CrossRef Medline

López-Bendito G, Molnár Z (2003) Thalamocortical development: how are we going to get there? Nat Rev Neurosci 4:276-289. CrossRef Medline

López-Bendito G, Sánchez-Alcañiz JA, Pla R, Borrell V, Picó E, Valdeolmillos M, Marín O (2008) Chemokine signaling controls intracortical migration and final distribution of GABAergic interneurons. J Neurosci 28 : 1613-1624. CrossRef Medline

Lysko DE, Putt M, Golden JA (2011) SDF1 regulates leading process branching and speed of migrating interneurons. J Neurosci 31:1739_ 1745. CrossRef Medline

Martínez-Cerdeño V, Noctor SC, Kriegstein AR (2006) The role of intermediate progenitor cells in the evolutionary expansion of the cerebral cortex. Cereb Cortex 16:1152-161. CrossRef Medline

Mire E, Mezzera C, Leyva-Díaz E, Paternain AV, Squarzoni P, Bluy L, Castillo-Paterna M, López MJ, Peregrín S, Tessier-Lavigne M, Garel S, Galcerán J, Lerma J, López-Bendito G (2012) Spontaneous activity regulates Robol transcription to mediate a switch in thalamocortical axon growth. Nat Neurosci 15:1134-1143. CrossRef Medline

Molnár Z, Adams R, Goffinet AM, Blakemore C (1998) The role of the first postmitotic cortical cells in the development of thalamocortical innervation in the reeler mouse. J Neurosci 18:5746-5765. Medline

Mueller W, Schütz D, Nagel F, Schulz S, Stumm R (2013) Hierarchical organization of multi-site phosphorylation at the CXCR4 C terminus. PloS one 8:e64975. CrossRef Medline

Nagasawa T, Hirota S, Tachibana K, Takakura N, Nishikawa S, Kitamura Y, Yoshida N, Kikutani H, Kishimoto T (1996) Defects of B-cell lymphopoiesis and bone-marrow myelopoiesis in mice lacking the CXC chemokine PBSF/SDF-1. Nature 382:635-638. CrossRef Medline

Parnavelas JG (2000) The origin and migration of cortical neurones: new vistas. Trends Neurosci 23:126-131. CrossRef Medline

Price DJ, Clegg J, Duocastella XO, Willshaw D, Pratt T (2012) The importance of combinatorial gene expression in early mammalian thalamic patterning and thalamocortical axonal guidance. Front Neurosci 6:37. CrossRef Medline

Pujol F, Kitabgi P, Boudin H (2005) The chemokine SDF-1 differentially 
regulates axonal elongation and branching in hippocampal neurons. J Cell Sci 118:1071-1080. CrossRef Medline

Rubenstein JL (2011) Annual research review: development of the cerebral cortex: implications for neurodevelopmental disorders. J Child Psychol Psychiatry 52:339-355. CrossRef Medline

Sánchez-Alcañiz JA, Haege S, Mueller W, Pla R, Mackay F, Schulz S, LópezBendito G, Stumm R, Marín O (2011) Cxcr7 controls neuronal migration by regulating chemokine responsiveness. Neuron 69:77-90. CrossRef Medline

Sessa A, Mao CA, Colasante G, Nini A, Klein WH, Broccoli V (2010) Tbr2positive intermediate (basal) neuronal progenitors safeguard cerebral cortex expansion by controlling amplification of pallial glutamatergic neurons and attraction of subpallial GABAergic interneurons. Genes Dev 24:1816-1826. CrossRef Medline

Stumm R, Höllt V (2007) CXC chemokine receptor 4 regulates neuronal migration and axonal pathfinding in the developing nervous system: implications for neuronal regeneration in the adult brain. J Mol Endocrinol 38:377-382. CrossRef Medline

Stumm RK, Rummel J, Junker V, Culmsee C, Pfeiffer M, Krieglstein J, Höllt V, Schulz S (2002) A dual role for the SDF-1/CXCR4 chemokine receptor system in adult brain: isoform-selective regulation of SDF-1 expression modulates CXCR4-dependent neuronal plasticity and cerebral leukocyte recruitment after focal ischemia. J Neurosci 22:5865-5878. Medline

Stumm RK, Zhou C, Ara T, Lazarini F, Dubois-Dalcq M, Nagasawa T, Höllt $\mathrm{V}$, Schulz S (2003) CXCR4 regulates interneuron migration in the developing neocortex. J Neurosci 23:5123-5130. Medline

Stumm R, Kolodziej A, Schulz S, Kohtz JD, Höllt V (2007) Patterns of SDFlalpha and SDF-1gamma mRNAs, migration pathways, and phenotypes of CXCR4-expressing neurons in the developing rat telencephalon. J Comp Neurol 502:382-399. CrossRef Medline
Suzuki-Hirano A, Ogawa M, Kataoka A, Yoshida AC, Itoh D, Ueno M, Blackshaw S, Shimogori T (2011) Dynamic spatiotemporal gene expression in embryonic mouse thalamus. J Comp Neurol 519:528-543. CrossRef Medline

Tanaka DH, Yanagida M, Zhu Y, Mikami S, Nagasawa T, Miyazaki J, Yanagawa Y, Obata K, Murakami F (2009) Random walk behavior of migrating cortical interneurons in the marginal zone: time-lapse analysis in flat-mount cortex. J Neurosci 29:1300-1311. CrossRef Medline

Tiveron MC, Rossel M, Moepps B, Zhang YL, Seidenfaden R, Favor J, König N, Cremer H (2006) Molecular interaction between projection neuron precursors and invading interneurons via stromal-derived factor 1 (CXCL12)/CXCR4 signaling in the cortical subventricular zone/intermediate zone. J Neurosci 26:13273-13278. CrossRef Medline

Tzeng YS, Li H, Kang YL, Chen WC, Cheng WC, Lai DM (2011) Loss of Cxcl12/Sdf- 1 in adult mice decreases the quiescent state of hematopoietic stem/progenitor cells and alters the pattern of hematopoietic regeneration after myelosuppression. Blood 117:429-439. CrossRef Medline

Vasistha NA, Garcia-Moreno F, Arora S, Cheung AF, Arnold SJ, Robertson EJ, Molnár Z (2014) Cortical and clonal contribution of Tbr2 expressing progenitors in the developing mouse brain. Cereb Cortex. Advance online publication. Retrieved June 13, 2014. CrossRef Medline

Vue TY, Aaker J, Taniguchi A, Kazemzadeh C, Skidmore JM, Martin DM, Martin JF, Treier M, Nakagawa Y (2007) Characterization of progenitor domains in the developing mouse thalamus. J Comp Neurol 505:73-91. CrossRef Medline

Wang Y, Li G, Stanco A, Long JE, Crawford D, Potter GB, Pleasure SJ, Behrens T, Rubenstein JL (2011) CXCR4 and CXCR7 have distinct functions in regulating interneuron migration. Neuron 69:61-76. CrossRef Medline

Wonders CP, Anderson SA (2006) The origin and specification of cortical interneurons. Nat Rev Neurosci 7:687-696. CrossRef Medline 